Pacific Journal of Mathematics

NONEXISTENCE OF CERTAIN CLOSED COMPLEX
GEODESICS IN THE MODULI SPACE OF CURVE 


\title{
NON-EXISTENCE OF CERTAIN CLOSED COMPLEX GEODESICS IN THE MODULI SPACE OF CURVES
}

\section{DOMINGO TOLEDO}

\begin{abstract}
We prove that most compact totally geodesic curves in the Siegel moduli space $A_{g}$ of $g$-dimensional principally polarized abelian varieties cannot lie in the image of the period mapping of the moduli space $M_{g}$ of smooth curves of genus $g$. The meaning of "most" is in terms of the holomorphic sectional curvature of Siegel space-see the precise statement below.
\end{abstract}

The reason for studying this question is that it gives some idea of the differential geometry of the period mapping $M_{g} \rightarrow A_{g}$. This mapping is presumably rather curved, i.e., the image of $M_{g}$ in $A_{g}$ is curved relative to the locally symmetric geometry of $A_{g}$. The best way to make this precise would be to compute the second fundamental form of the period mapping. This could be an involved computation, perhaps not immediately interpretable in geometric terms. Hence we prefer first to take a more elementary approach and ask if the image of $M_{g}$ contains any straight lines of the symmetric geometry, i.e., any complex totally geodesic curves. The easiest question to decide, and the only one studied here, is whether any closed complex geodesics lie in the image. The question of geodesics of finite area is quite interesting, but more difficult.

We study this question by applying the Miyaoka inequality [5] to the complex surface induced by the curve in $M_{g}$. It gives that the image of the classifying mapping for the cohomology of the fibers (period mapping) has area less than $1 / 3$ of the expected maximum for the area of a mapping into the period space. This strongly suggests curvature properties of the period mapping.

To show that the above restrictions are not vacuous, we point out in $\$ 3$ that closed geodesics violating the restrictions do exist in $A_{g}$. These are constructed from classical examples of Hilbert modular surfaces in $A_{2}$. We also remark that, for $g \geq 3, M_{g}$ has plenty of compact curves. Examples starting in $g=6$ are explicitly constructed in $[1,4]$.

A final algebro-geometric remark is that complex geodesics in $A_{g}$ are related to reducibility of the monodromy representation. Namely a complex geodesic of curvature $-1 / l$ (cf. $\$ 1$ ) parametrizes a family of abelian 
varieties with a fixed $(g-l)$-dimensional abelian subvariety. This is a necessary, but not sufficient, condition for a curve in $A_{g}$ to be geodesic. The examples in $[\mathbf{1}, \mathbf{4}]$ have a fixed part in their monodromy representation but are not geodesic.

I am grateful to J. Carlson and $\mathrm{H}$. Clemens for discussions on these topics, and to the referee for suggesting a considerable improvement on the presentation.

1. Statement and proof of the theorem. Let $H_{g}$ be the Siegel upper half plane of genus $g$, i.e., the space of complex symmetric $g$ by $g$ matrices with positive definite imaginary part. It will also be convenient to look at its realization as a bounded domain, namely

$\left\{Z: Z\right.$ a complex $g$ by $g$ matrix, $\left.Z^{t}=Z, 1-Z^{*} Z>0\right\}$.

If we let

$$
\rho=-\log \operatorname{det}\left(1-Z^{*} Z\right),
$$

then $\omega=d d \mathbf{C}_{\rho}$ is the Kähler form of the Bergmann metric of $H_{g}$, and its holomorphic sectional curvature function has for image the interval $[-1,-1 / g]$. Let $\Delta^{g} \subset H_{g}$ be the submanifold of diagonal matrices $\operatorname{diag}\left\{z_{1}, \ldots, z_{g}\right\},\left|z_{i}\right|<1$. Then $\Delta^{g}$ is a maximal totally geodesic polydisk and the totally geodesic complex curves in $H_{g}$ are equivalent, under the group of isometries, to one of the curves

$$
z \rightarrow \operatorname{diag}\{(z, \ldots, z, 0, \ldots, 0)\}, \quad|z|<1,
$$

where the number $l$ of non-zero entries takes the values $l=1, \ldots, g$. Since each factor of $\Delta^{g}$ has the Poincaré metric of constant curvature -1 , a geodesic of type $l$ has a metric of constant curvature $-1 / l$.

We can think of $H_{g}$ as the subset of the Grassmannian $G(g, 2 g)$ of complex $g$-planes in $\mathbf{C}^{2 g}=\left\{(u, v): u=\left(u_{1}, \ldots, u_{g}\right), v=\left(v_{1}, \ldots, v_{g}\right)\right\}$ on which the skew form $\Omega=d u_{1} \wedge d v_{1}+\cdots+d u_{g} \wedge d v_{g}$ vanishes and the Hermitian form $h=|u|^{2}-|v|^{2}$ is positive definite, the correspondence given by $Z \rightarrow\left\{(u, Z u): u \in \mathbf{C}^{g}\right\}$. Let $E$ be the restriction of the tautological subbundle over $G(g, 2 g)$ to $H_{g}$. Then the form $|u|^{2}-|v|^{2}$ gives an invariant Hermitian metric on $E$, whose matrix relative to the frame $\left(e_{i}, Z e_{i}\right), e_{i}=(0, \ldots, 1, \ldots, 0)$, is precisely $1-Z^{*} Z$. Hence $c_{1}(E)$ is represented by the form

$$
\begin{aligned}
\frac{i}{2 \pi} d^{\prime \prime} d^{\prime} \log \operatorname{det}\left(1-Z^{*} Z\right) & =\frac{d d^{\mathbf{C}}}{4 \pi}\left(-\log \operatorname{det}\left(1-Z^{*} Z\right)\right) \\
& =\frac{1}{4 \pi} d d^{\mathbf{C}} \rho=\frac{1}{4 \pi} \omega .
\end{aligned}
$$


Thus

$$
c_{1}(E)=\frac{1}{4 \pi} \omega
$$

Now let $\Gamma$ be a torsion-free subgroup of finite index in the integral symplectic group and let $\tilde{A}_{g}=\Gamma \backslash H_{g}$. Suppose $C$ is a smooth compact algebraic curve of genus $h$ and $f: \mathbf{C} \rightarrow \tilde{A}_{g}$ is a map whose image is a totally geodesic curve of curvature $-1 / l$. Then the Gauss-Bonnet formula gives

$$
\int_{C} c_{1}\left(f^{*} E\right)=l(h-1),
$$

because $c_{1}\left(f^{*} E\right)=(1 / 4 \pi) f^{*} \omega$ and $f^{*} \omega$ is the area form of a metric of constant curvature $-1 / l$ on $C$.

Finally, let $\Phi: M_{g} \rightarrow A_{g}$ be the period mapping, and let $\tilde{M}_{g}$ be a finite covering of $M_{g}$ so that $\Phi$ lifts to a map $\phi: \tilde{M}_{g} \rightarrow \tilde{A}_{g}$.

THEOREM. Let $C \subset \tilde{M}_{g}$ be a smooth, compact curve of genus $h$ so that $\Phi(C)$ is a totally geodesic curve in $\tilde{A}_{g}$ of curvature $-1 / l$. Then $l \leq$ $(g-1) / 3$.

Proof. Over $\tilde{M}_{g}$ we have a universal curve whose restriction to $C$ gives a surface $X$ and a fibration $\pi: X \rightarrow C$ whose fibers are smooth curves of genus $g$. We compute the Chern numbers of $X$. First, $c_{2}(X)=$ $c_{1}(C) c_{1}$ (fibre) $=4(h-1)(g-1)$. To compute $c_{1}^{2}(X)$ we use the Grothendieck-Riemann-Roch formula:

$$
\pi_{*}(\operatorname{Todd}(X))=\operatorname{Todd}(C)\left(\operatorname{ch}\left(R^{0} \pi_{*} O_{X}\right)-\operatorname{ch}\left(R^{1} \pi_{*} O_{X}\right)\right) .
$$

Note that $R^{1} \pi_{*} O_{X}$ is the locally free sheaf on $C$ corresponding to the vector bundle $f^{*} E^{*}$, where $f=\Phi \mid C$. Thus $\operatorname{ch}^{0}\left(R^{1} \pi_{*} O_{X}\right)=g$ and $\operatorname{ch}^{1}\left(R^{1} \pi_{*} O_{X}\right)=c_{1}\left(f^{*} E^{*}\right)=-c_{1}\left(f^{*} E\right)=-l(h-1)$, by (2). Thus we can evaluate (3) on the fundamental cycle of $C$ and obtain

$$
\frac{c_{1}^{2}(X)+c_{2}(X)}{12}=(1-h)(1-g)+l(h-1) .
$$

Substituting $c_{2}(X)=4(h-1)(g-1)$ and solving for $c_{1}^{2}(X)$ we get

$$
c_{1}^{2}(X)=4(h-1)(2 g-2+3 l) \text {. }
$$

Since $X$ is a minimal surface of general type, Miyaoka's inequality $c_{1}^{2}(X) \leq 3 c_{2}(X)$ gives $l \leq g-1 / 3$, as was to be proved. 
REMARKs (1) We think it is very unlikely that any closed complex geodesics in $A_{g}$ can lie in the image of $M_{g}$, but, except for the present case, can only rule out the case $l=1$, see $\$ 2$.

(2) We stated the theorem in terms of non-existence of certain closed geodesics in order to give a concrete geometric statement. But we could equally well have stated the result as an estimate on $\left|\operatorname{ch}^{1}\left(R^{1} \pi_{*} O_{X}\right)\right|$ for any fibration $\pi: X \rightarrow C$ with fibres smooth curves of genus $g$. Namely, the same argument gives the inequality

$$
\left|\operatorname{ch}^{1}\left(R^{1} \pi_{*} O_{X}\right)\right| \leq \frac{1}{3}(g-1)(h-1) .
$$

It is interesting to rewrite this in terms of $\omega$, using (2), i.e.

$$
\left|\int_{C} f^{*} \omega\right| \leq \frac{4 \pi}{3}(g-1)(h-1),
$$

and then to contrast this with the sharp estimate for mappings $f: C \rightarrow \tilde{A}_{g}$ as in [2]:

$$
\left|\int_{C} f^{*} \omega\right| \leq 4 \pi g(h-1) .
$$

Thus the area of a mapping $f: C \rightarrow \tilde{A}_{g}$ that factors through $M_{g}$ is less than $1 / 3$ of the expected maximum.

2. Geodesics of curvature -1 . The purpose of this section is to show that closed geodesics of curvature - 1 (i.e. $l=1$ ) do not exist in $A_{g}$. It will be convenient to work with the usual upper half plane model of $H_{g}$ and regard it as the subset of the Grassmannian $G(g, 2 g)$ of $p$-planes in $\mathrm{C}^{2 g}$ on which the skew form $\Omega$ as in $\$ 1$ vanishes and the Hermitian form

$$
h=i \sum u_{j} \bar{v}_{j}-v_{j} \bar{u}_{j}
$$

is positive definite, the correspondence given as in $\$ 1$ by assigning to a matrix its graph. A geodesic of curvature -1 is the image under some element of the real symplectic group of the curve

$$
z \rightarrow \operatorname{diag}(z, i, \ldots, i), \quad z \in H_{1} .
$$

From this it is clear that a geodesic $L$ of curvature -1 is characterized in the Grassmannian as the set of all subspaces

$$
L=\left\{W \subset \mathbf{C}^{2 g}: \operatorname{dim} W=g, W \supset V, h / W>0\right\}
$$

for some fixed subspace $V$ of $\mathbf{C}^{2 g}, \operatorname{dim} V=g-1$.

If $X$ is a subspace of $\mathbf{C}^{2 g}$, let $X^{\perp}, X^{0}$ denote its orthogonal complement with respect to $h, \Omega$ respectively. Given $L$ as above, choose a basis $e_{1}, \ldots, e_{2 g}$ for $\mathbf{C}^{2 g}$ as follows: $e_{2}, \ldots, e_{g}$ a basis for $V, e_{g+2}, \ldots, e_{2 g}$ 
a basis for $\left(V^{0}\right)^{\perp}, e_{1}, e_{g}$ a basis for $Y^{\perp}$, where $Y=V \oplus\left(V^{0}\right)^{\perp}$, so that $h\left(e_{1}\right)>0$ and $h\left(e_{g}\right)<0$.

Suppose $\Gamma \subset \operatorname{Sp}(g, Z)$ stabilizes $L$. Then relative to a basis as above the elements of $\Gamma$ have matrices of the form

$$
\left(\begin{array}{cccc}
a & 0 & b & 0 \\
0 & A & 0 & 0 \\
c & 0 & d & 0 \\
0 & 0 & 0 & A^{-1}
\end{array}\right), \quad\left(\begin{array}{ll}
a & b \\
c & d
\end{array}\right) \in \mathrm{Sp}(1, I R), \quad A \in U(g-1) .
$$

Since $\Gamma$ is a group we must have that all the matrices $A=A(\gamma), \gamma \in \Gamma$, must commute. Thus if the complex geodesic $\Gamma \backslash L$ were compact, $\Gamma$ would be non-abelian, hence there is a subgroup $\Gamma^{\prime} \subset \Gamma$, different from the identity, so that $A(\gamma)=$ id for all $\gamma \in \Gamma^{\prime}$. The compactness of $\Gamma \backslash L$ also implies that all elements of $\Gamma$ give hyperbolic motions of the hyperbolic plane, so the 2 by 2 sub-block in the above matrix cannot have one as an eigenvalue if $\gamma \neq$ id. Thus $Y$ is the eigenspace corresponding to the eigenvalue one of a matrix with integral entries, hence defined over the integers. $Y^{\perp}$ is then defined over $\mathbf{Z}$, so the basis $e_{1}, \ldots, e_{2 g}$ can be chosen so that all the entries of the above block and the complementary block are integral for all $\gamma \in \Gamma$. From this and the compactness of $U(g-1)$ it follows easily that the action of $\Gamma$ on $L$ is commensurable with the usual action of $\operatorname{Sp}(1, \mathbf{Z})=\operatorname{SL}(2, \mathbf{Z})$ on the upper half plane $H_{1}$, which does not have compact quotient. Thus no complex geodesic of curvature -1 can be compact in $A_{g}$.

REMARK. The same argument shows that if $\Gamma \subset \operatorname{Sp}(g, \mathbf{Z})$ is the stabilizer of a complex geodesic of curvature -1 , then either 1) $\Gamma=\{$ id $\}$, 2) $\Gamma=\mathbf{Z}$, or 3$) \Gamma$ is commensurable with $\operatorname{SL}(2, \mathbf{Z})$. The latter case classifies all such geodesics of finite area. Geometrically they parametrize families of $g$-dimensional abelian varieties with a fixed $(g-1)$ dimensional abelian subvariety. An analogous classification can be similarly obtained for certain totally geodesic embeddings of $H_{l}$ in $H_{g}$, $1<l<g$.

3. Closed complex geodesics in Siegel space. Finally we remark that closed complex geodesics that violate the inequality of $\$ 1$ do exist in $A_{g}$. For $g=2$ and $l=2$ the construction is based on the classical embeddings of Hilbert modular surfaces in $A_{2}$ studied by Hecke. We give [3], Chapter 7 as a convenient reference. Namely, if $p$ is a prime of the form $4 k+1$, there is an embedding of the Hilbert modular surface $\mathrm{SL}(2, O) \backslash H \times H$ in $A_{2}$, where $O$ is the ring of integers in $\mathbf{Q}(\sqrt{p})$. The 
image of this embedding is covered by a totally geodesic $\mathrm{H} \times \mathrm{H}$ in $\mathrm{H}_{2}$. Now the Hilbert modular surface contains compact geodesic curves of curvature $-1 / 2$; these are the Shimura curves discussed in $[3,6 \mathrm{e}]$. Their image in $A_{2}$ are the desired geodesics.

Now for any $k \leq[g / 2]$ we have a natural embedding $A_{2}^{k} \rightarrow A_{g}$ induced by the mapping $H_{2}^{k} \rightarrow H_{g}$ given by

$$
Z_{1}, \ldots, Z_{k} \rightarrow \text { block diagonal }\left(Z_{1}, \ldots, Z_{k}\right) \text {. }
$$

The diagonal images of the above examples in $A_{2}$ give closed complex geodesics of curvature $-1 / 2 k$.

\section{REFERENCES}

[1] M. F. Atiyah, The signature of fibre bundles, in Global Analysis, Princeton Math. Series 29, Princeton University Press, 1969.

[2] A. Domic and D. Toledo, The Gromov norm of the Käehler class of symmetric domains, Math. Annalen, 1987.

[3] F. Hirzebruch and G. van der Geer, Lectures on Hilbert Modular Surfaces, Presses de l'Univ. de Montreal, 1981.

[4] K. Kodaira, A certain type of irregular algebraic surface, J. Analyse Math., 19 (1967), 207-215.

[5] Y. Miyaoka, On the Chern numbers of surfaces of general type, Invent. Math., 42 (1977), 225-237.

Received November 13, 1985. Supported by the Sloan Foundation and The National Science Foundation.

UNIVERSITY OF UTAH

Salt Lake City, UT 84112 


\section{PACIFIC JOURNAL OF MATHEMATICS EDITORS}

V. S. VARADARAJAN

(Managing Editor)

University of California

Los Angeles, CA 90024

Herbert Clemens

University of Utah

Salt Lake City, UT 84112

R. FINN

Stanford University

Stanford, CA 94305
ROBION KIRBY

University of California

Berkeley, CA 94720

C. C. MOORE

University of California

Berkeley, CA 94720

HAROLD STARK

University of California, San Diego La Jolla, CA 92093

\section{ASSOCIATE EDITORS}
R. ARENS
E. F. BECKENBACH
B. H. NEUMANN
F. WOLF
K. YosHIDA (1906-1982)

\section{SUPPORTING INSTITUTIONS}

UNIVERSITY OF ARIZONA

UNIVERSITY OF BRITISH COLUMBIA

CALIFORNIA INSTITUTE OF TECHNOLOGY

UNIVERSITY OF CALIFORNIA

MONTANA STATE UNIVERSITY

UNIVERSITY OF NEVADA, RENO

NEW MEXICO STATE UNIVERSITY

OREGON STATE UNIVERSITY
UNIVERSITY OF OREGON UNIVERSITY OF SOUTHERN CALIFORNIA

STANFORD UNIVERSITY

UNIVERSITY OF HAWAII

UNIVERSITY OF TOKYO

UNIVERSITY OF UTAH

WASHINGTON STATE UNIVERSITY

UNIVERSITY OF WASHINGTON 


\section{Pacific Journal of Mathematics}

Vol. 129, No. $1 \quad$ May, 1987

Aldridge Knight Bousfield, Uniqueness of infinite deloopings for

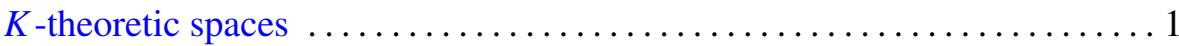

Mark Gregory Davidson, The harmonic representation of $U(p, q)$ and its

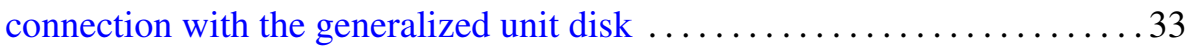

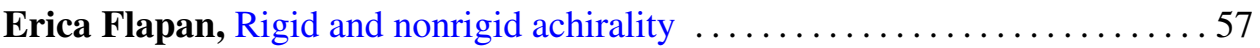

Peter Abraham Greenberg, Pseudogroups of $C^{1}$ piecewise projective

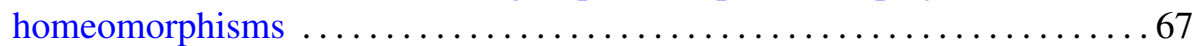

Peter Martin Knopf, Maximal functions on the unit $n$-sphere $\ldots \ldots \ldots \ldots 77$

Norman Jay Levitt and Andrew Ranicki, Intrinsic transversality

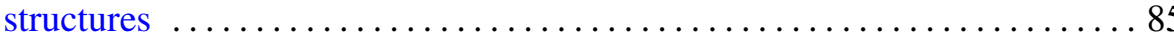

Susan Szczepanski, Invariant submanifolds of free cyclic actions on

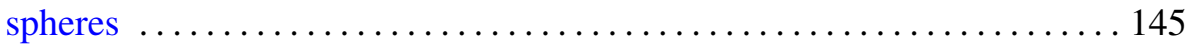

Kazimierz Szymiczek, Generalized rigid elements in fields . . . . . . . . 171

Domingo Toledo, Nonexistence of certain closed complex geodesics in the

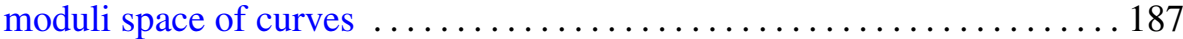

Graham H. Williams, The best modulus of continuity for solutions of the

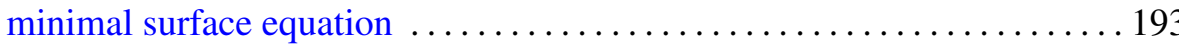

